\title{
Relação da massa e força muscular com nível de atividade física de usuários de Unidades Básicas de Saúde
}

\author{
Relation of the muscle mass and strength with low level of physical \\ activity in users of Public Health Basic Units
}

Vanessa Ribeiro dos Santos 1,3 , Daiane de Oliveira Macedo ${ }^{3}$, Camila Bispo Nascimento 3 , Diego Giulliano Destro Christofaro ${ }^{1,2}$, Luís Alberto Gobbo 1,2,3

\section{Resumo}

O objetivo do presente estudo foi analisar a relação da massa muscular (MM) e força muscular (FM) com nível de atividade física de usuários de Unidades Básicas de Saúde (UBS) de Presidente Prudente, SP. A amostra foi composta por 474 homens e mulheres com idade $\geq 50$ anos. Como identificador da MM e FM, utilizou-se o índice de massa muscular (IMM), e a dinamometria manual, respectivamente. A atividade física habitual (AFH) foi estimada por meio de questionário Baecke. Para classificar a amostra (baixo IMM, baixa FM e atividade física), utilizou-se valores abaixo do tercil 1 de cada variável, segundo sexo. O teste qui-quadrado e a análise de regressão logística foram utilizados para análise das associações. Observou-se que o baixo nível de atividade física ocupacional associou-se a baixa FM $\left(\chi^{2}=5,859 ; \mathrm{p}=0,015\right)$, em homens $\left(\chi^{2}=5,907\right.$; $\mathrm{p}=0,015)$. Foi verificado também que o baixo nível de atividade física no domínio exercício físico no lazer associou-se a baixa FM $\left(\chi^{2}=9,585 ; p=0,002\right)$ em mulheres $\left(\chi^{2}=13,191 ; p \leq 0,001\right)$. Foi verificada associação positiva entre baixa FM e baixo nível de AFH para a amostra total $\left(\chi^{2=6,385 ;} \mathrm{p}=0,012\right)$ e em homens $\left(\chi^{2}=4,938 ; \mathrm{p}=0,026\right)$. Pessoas com baixo nível de AFH apresentaram 1,89 maiores chances (IC95\% 1,26-2,84) de terem baixa FM, sendo que homens apresentaram 2,76 mais chances (IC95\% 1,33-5,73). Em conclusão, pessoas com baixo nível de atividade física apresentam maior chance de terem baixa FM, em especial, os homens.

\section{Palavras-chave}

Atividade motora; Sarcopenia; Dinamômetro de força muscular; Antropometria.

\begin{abstract}
The aim of this study was to analyze the relation of muscle mass (MM) and strength (MS) with physical activity level of users of Public Basic Health Units of Presidente Prudente, SP. The sample consisted of 474 men and women aged over 50 years. As identifiers of MM and MS, we used the muscle mass index (MMI), and handgrip strength, respectively. Habitual physical activity (HPA) was measured by Baecke questionnaire. To categorize the sample (low MMI, MS and physical active), was used values below 25th percentile of each variable, according to sex. The chi-square test and logistic regression analysis were used to analyze the associations. It was observed that low level of occupational physical activity was associated with low SM $\left(\chi^{2}=5.859 ; p=0.015\right)$, in men $(\chi 2=5.907$; $p=0.015)$. Also found that low level of physical activity in the exercise at leisure was associated with low SM $(\chi 2=9.585 ; p=0.002)$, in women $(\chi 2=13.191 ; p \leq 0,001)$. Positive association was found between low SM and low HPA in total sample $(\chi 2=6.385 ; p=0.012)$ and men $(\chi 2=4.938 ; p=0.026)$. People with low level of HPA had 1.89 odds (CI95\% 1.26-2.84) more likely to have low SM, and men had 2.76 more chances (CI95\% 1.33-5.73). In conclusion, people with low level of physical activity are more likely to have low FM, especially men.
\end{abstract}

\section{Keywords}

Motor Activity; Sarcopenia; Muscle Strength Dynamometer; Anthropometry.
1 Programa de Pós-graduação em Ciências da Motricidade, Instituto de Biociências, Universidade Estadual Paulista, Rio Claro, São Paulo, Brasil

2 Departamento de Educação Física, Faculdade de Ciências e Tecnologia, Universidade Estadual Paulista, Presidente Prudente, São Paulo, Brasil

3 Laboratório de Avaliação do Sistema Musculoesquelético (LABSIM), Departamento de Educação Física, Faculdade de Ciências e Tecnologia, Universidade Estadual Paulista, Presidente Prudente, São Paulo, Brasil

\section{Introdução}

A expectativa de vida no mundo está aumentando, sobretudo em decorrência da redução das taxas de mortalidade e fecundidade ${ }^{1}$. Com isso, cresce também a preocupação e os cuidados com a saúde de indivíduos 
mais velhos, que apresentam diversos fatores de risco, entre eles o declínio progressivo na quantidade (massa) e qualidade (função) do tecido muscular ${ }^{2,3,4,5}$.

Dentre as várias funções prejudicadas pelo declínio da força (FM) e massa muscular (MM), estão presentes as deficiências ocorridas nos sistemas motor e sensorial, podendo levar a distúrbios em relação à postura e a redução do equilíbrio ${ }^{6}$. O comprometimento desses sistemas dificulta a realização de tarefas cotidianas. Ademais essa redução aumenta a possibilidade para quedas e fraturas, resultando posteriormente na incapacidade e dependência desses indivíduos, afetando a própria qualidade de vida e de seus familiares?

Em grande parte a manutenção da FM e MM, bem como da funcionalidade de cada indivíduo não está associada somente à sua idade cronológica, existem diferentes tipos de comportamento de estilo de vida, como por exemplo, a prática de atividade física, que quando realizados regularmente no decorrer da vida amenizam e tardam as consequências inevitáveis do processo de envelhecimento ${ }^{8}$. Contudo, apesar dos conhecidos benefícios da pratica regular de atividade física para a saúde muscular, a prática e aderência da população brasileira adulta por tais atividades são baixas (14,8\% no lazer, $42,8 \%$ no trabalho, $11,7 \%$ no deslocamento e 48,5\% nas atividades domésticas) ${ }^{9}$, especialmente, em idades mais avançadas ${ }^{9,10}$.

Dessa forma, o conhecimento referente a qual dos elementos (MM ou FM) é mais afetado pela prática insuficiente de atividade física em diferentes domínios, aspectos esses pouco explorado na literatura, torna-se importante para direcionar os profissionais da saúde na promoção de programas de intervenção, especialmente na população atendida em Unidades Básicas de Saúde, que em sua maioria é composta por indivíduos mais velhos e mais susceptíveis ao desenvolvimento de agravos $^{11}$. Com isso, o objetivo do presente estudo foi analisar a relação da MM e FM com nível de atividades físicas de usuários de Unidades Básicas de Saúde de Presidente Prudente, SP.

\section{Métodos}

\section{Amostra}

A amostra foi composta por 474 homens e mulheres com idade igual ou superior a 50 anos usuários das duas Unidades Básicas de Saúde (UBS) com maior número de pacientes cadastrados e fluxo de atendimentos dentre as 15 UBS e as 11 Estratégias de Saúde da Família do município de Presidente Prudente, SP.

Os Critérios de inclusão foram: i) ter idade igual ou superior a 50 anos ii) ser cadastrado e morar próximo a UBS. Foram excluídos do estudo indivíduos que apresentavam agravos articulares nos membros superiores que impossibilitasse a realização do teste de força muscular ou possuísse alguma doença que propicie redução da massa muscular como: câncer, HIV/Aids, tuberculose e doença renal crônica.

Os pacientes foram abordados no momento que chegavam às UBS, para atendimento médico ou retirada de medicamentos, por alunos do curso de Educação Física, previamente treinados. Os procedimentos de condução da pesquisa foram esclarecidos aos pacientes e, quando o voluntário consentia na participação, respondia às entrevistas e avaliações. A pesquisa foi realizada no período da manhã e tarde para maior abrangência de população.

O estudo foi aprovado pelo Comitê de Ética em Pesquisa envolvendo seres humanos da Universidade Estadual Paulista "Julio de Mesquita Filho", Campus de Presidente Prudente (Processo 241.291/2013). 


\section{Variáveis do estudo}

\section{- Massa Muscular}

Como identificador da MM, utilizou-se o índice de massa muscular (IMM), a partir da razão da MM pela altura, em metros, ao quadrado. A MM foi calculada mediante utilização de equação preditiva proposta por Lee et al. ${ }^{12}$. Para tanto, foram registrados dados de sexo (masculino $=1$, feminino $=0$ ), idade (em anos), e etnia (branca $=0$, negra $=1,4$, asiático $=-1,2$ ), além de medidas de peso, em $\mathrm{kg}$, e estatura, em $\mathrm{cm}$, seguindo as recomendações de Lohman et $\mathrm{al}^{13}$.

\section{- Força Muscular}

Dinamometria de preensão manual foi utilizada para avaliação da FM. Para tanto, dinamômetro da marca Camry, modelo EH101 (Guangdong, China), foi utilizado. Os participantes permaneceram na posição sentada, com os cotovelos flexionados em 90 graus, junto à cintura. As medidas foram realizadas nos dois membros superiores, em duplicata, de forma rotacional, sendo registrado o maior valor para cada membro. Para fins de análise, utilizou-se os valores do braço dominante do avaliado, em kg.

\section{- Atividades Física Habitual}

A prática de atividade física habitual (AFH) foi verificada por meio de entrevista face a face, utilizando o questionário desenvolvido por Baecke et al. ${ }^{14}$, traduzido e validado para a população brasileira por Florindo et al. ${ }^{15} \mathrm{com}$ uma população com idade média de 62,5 anos.

O instrumento é composto por 16 questões e investiga o nível de AFH em três domínios: ocupacional (tipo de ocupação e atividades realizadas durante o trabalho); exercício físico no lazer (prática de exercícios físicos regulares); e atividades de lazer e locomoção (assistir TV, caminhar ou andar de bicicleta no lazer e para locomoção). A partir da aplicação do questionário, foi possível identificar o nível de atividade física em cada domínio. A soma dos escores de cada seção representa o escore total, ou seja, a AFH.

Para classificação da AFH foi utilizada a fórmula proposta por Baecke et al. ${ }^{14}$ considerando o somatório dos diferentes domínios da prática de atividade física. $\mathrm{O}$ instrumento fornece um escore adimensional sem unidade de medida e quanto maior esse escore, maior a prática de atividade física. Posteriormente, a amostra foi subdividida em tercis segundo cada domínio (ocupacional, exercício físico no lazer e, atividade física no lazer e locomoção) e AFH. Os indivíduos avaliados foram estratificados da seguinte forma: tercil 1 - mais baixo nível de atividade física; tercis 2 e 3 mais alto nível de atividade física.

\section{Análise estatística}

A normalidade dos dados foi testada pelo teste Kolmogorov Smirnov. A estatística descritiva das variáveis numéricas foi composta por valores de mediana e intervalo interquartil. Para os dados categóricos, valores percentuais. Para as variáveis categóricas, o teste qui-quadrado foi empregado para indicar associações entre as variáveis dependentes (MM e FM) e independente (AFH). As variáveis associadas com a variável dependente foram inseridas no modelo multivariado construído pela análise de regressão logística binária, que expressa à magnitude das associações em valores de razão de chance (OR) e seus respectivos intervalos de confiança de $95 \%$. As análises estatísticas foram realizadas pelo programa Stata IC 11.0 e a significância estatística foi estabelecida em $5 \%$. 


\section{Resultados}

Para classificação dos sujeitos para MM (baixo IMM e normal), utilizou-se os pontos de corte baseados nos valores do menor tercil do IMM $\left(\mathrm{em} \mathrm{kg} / \mathrm{m}^{2}\right)$, segundo sexo, com valores de $10,11 \mathrm{~kg} / \mathrm{m}^{2}$ e $7,97 \mathrm{~kg} / \mathrm{m}^{2}$ para homens e mulheres, respectivamente.

Os participantes foram também divididos em duas categorias para FM (baixa FM e normal) com ponto de corte abaixo do menor tercil de FM, em kg, segundo sexo, com valores de $32,43 \mathrm{~kg}$ e $18,73 \mathrm{~kg}$ para homens e mulheres, respectivamente.

Os pontos de corte para atividade física nos domínios ocupacional, exercício físico no lazer e atividade física no lazer e locomoção e AFH foram para homens $1,75,1,50,2,00$ e 5,37, respectivamente, e para mulheres, 2,50, 1,25, 1,75 e 5,75 respectivamente.

As características descritivas da amostra estão apresentadas na Tabela 1. Os homens avaliados no estudo apresentaram valores medianos superiores $(p \leq 0,001)$ para as variáveis idade, peso corporal, estatura, FM (dinamometria), MM e IMM, bem como para o escore no domínio da atividade física no lazer e locomoção, enquanto as mulheres apresentaram maiores valores $(\mathrm{p} \leq 0,001)$ para as variáveis IMC e escore no domínio da atividade física ocupacional.

TABELA 1 - Valores de média e intervalo interquartil de variáveis descritivas dos participantes do grupo total e segundo sexo $(n=474)$.

\begin{tabular}{lccc}
\hline \multicolumn{1}{c}{ Variável } & Amostra Geral & Homens & Mulheres \\
\hline Idade (anos) & Mediana (P25-75) & Mediana $(\mathrm{P} 25-75)$ & Mediana (P25-75) \\
\hline AF Ocup (escore) & $61,3(55,8-68,3)$ & $65,5(57,5-71,7)^{*}$ & $60,2(55,2-66,7)$ \\
\hline AF Exerc (escore) & $2,6(2,1-3,0)$ & $2,1(0,1-2,7)^{*}$ & $2,7(2,4-3,0)$ \\
\hline AF Locom (escore) & $1,7(1,2-2,2)$ & $1,7(1,2-2,2)$ & $1,7(1,2-2,2)$ \\
\hline AFH (escore) & $2,0(1,7-2,2)$ & $2,2(1,7-2,7)^{*}$ & $2,0(1,5-2,5)$ \\
\hline Peso (kg) & $6,4(5,4-7,2)$ & $6,2(4,9-7,4)$ & $6,4(5,5-7,2)$ \\
\hline Estatura (cm) & $72,3(62,4-82,9)$ & $76,0(66,9-85,2)^{*}$ & $70,3(60,8-81,0)$ \\
\hline IMC (kg.m-2) & $156,5(151,6-163,6)$ & $167,0(161,1-171,0)^{*}$ & $154,0(149,3-158,0)$ \\
\hline Dinamometria (kg) & $28,7(25,6-32,8)$ & $27,8(25,2-30,3)^{*}$ & $29,5(25,9-34,2)$ \\
\hline MM (kg) & $24,0(19,0-31,7)$ & $35,9(30,4-42,1)^{*}$ & $21,1(17,6-25,2)$ \\
\hline IMM (kg.m-2) & $22,5(18,9-27,2)$ & $29,4(25,5-32,0)^{*}$ & $20,2(17,6-23,1)$ \\
\hline
\end{tabular}

Notas: AF Ocup=domínios da atividade física ocupacional; AF Exerc=domínio da atividade física no exercício físico no lazer; AF Locom=domínio da atividade física no lazer e locomoção; AFH=atividade física habitual; IMC=índice de massa corporal; $M M=$ massa muscular; IMM=índice de massa muscular; ${ }^{*}=$ diferenças entre sexos $p \leq 0,001$.

A Tabela 2 apresenta o nível de atividade física dos sujeitos em cada domínio. Foi verificada diferença significativa nas frequências entre sexos no domínio exercício físico no lazer, com as mulheres apresentando maior nível $(p<0,05)$. Observou-se que sujeitos com maior nível de atividade física ocupacional apresentaram melhor FM, especialmente, os homens. Foi verificado também que àqueles com maior nível de atividade física no domínio exercício físico no lazer também apresentaram melhor FM, nesse caso, especialmente as mulheres. Não foi observada associação entre a prática de atividade física do domínio lazer e locomoção e FM.

Não foi verificada associação entre a prática de atividade física em nenhum dos domínios e MM.

Quando os sujeitos foram categorizados segundo nível de AFH não foi verificado diferenças significativas nas frequências entre sexos para o nível de atividade física, seja 
para a amostra total, seja quando analisado grupos com MM baixa ou alta. Entretanto, na análise da FM, foi verificado para a amostra total e para pessoas do sexo masculino associação significativa entre baixa FM e menor nível de atividade física (Tabela 3).

TABELA 2 - Frequência dos participantes segundo nível de atividade física, em cada domínio, massa e força muscular para amostra geral e segundo o sexo.

\begin{tabular}{|c|c|c|c|c|c|c|}
\hline \multirow{4}{*}{ Categorias } & \multirow{2}{*}{\multicolumn{2}{|c|}{$\begin{array}{l}\text { Amostra geral } \\
\text { Nível de AFH }\end{array}$}} & \multirow{2}{*}{\multicolumn{2}{|c|}{$\begin{array}{c}\text { Homens } \\
\text { Nível de AFH }\end{array}$}} & \multirow{2}{*}{\multicolumn{2}{|c|}{$\begin{array}{c}\text { Mulheres } \\
\text { Nível de AFH }\end{array}$}} \\
\hline & & & & & & \\
\hline & Alto & Baixo & Alto & Baixo & Alto & Baixo \\
\hline & n (\%) & $n(\%)$ & $n(\%)$ & n (\%) & n (\%) & $n(\%)$ \\
\hline \multicolumn{7}{|c|}{ Atividade Física Ocupacional } \\
\hline Total & $334(70,5)$ & $140(29,5)$ & $102(69,4)$ & $42(30,6)$ & $234(70,9)$ & $96(29,1)$ \\
\hline $\mathrm{p}^{\mathrm{a}}$ & & & \multicolumn{4}{|c|}{0,748} \\
\hline \multicolumn{7}{|l|}{ MM } \\
\hline normal & $225(67,4)$ & $91(65,0)$ & $67(67,0)$ & $65,9(65,9)$ & $158(67,5)$ & $62(64,6)$ \\
\hline baixa & $109(32,6)$ & $49(35,0)$ & $33(33,0)$ & $34,1(34,1)$ & $76(32,5)$ & $34(35,4)$ \\
\hline$p^{b}$ & \multicolumn{2}{|l|}{0,618} & \multicolumn{2}{|c|}{0,898} & \multicolumn{2}{|c|}{0,607} \\
\hline \multicolumn{7}{|l|}{ Força } \\
\hline normal & $234(70,1)$ & $82(58,6)$ & $73(73,0)$ & $23(52,3)$ & $161(68,8)$ & $59(61,5)$ \\
\hline baixa & $100(29,9)$ & $58(41,4)$ & $27(27,0)$ & $21(47,7)$ & $73(31,2)$ & $37(38,5)$ \\
\hline$p^{b}$ & \multicolumn{2}{|l|}{$0,015^{*}$} & \multicolumn{2}{|c|}{$0,015^{*}$} & \multicolumn{2}{|c|}{0,199} \\
\hline \multicolumn{7}{|c|}{ Exercício Físico no Lazer } \\
\hline Total & $408(86,1)$ & $66(13,9)$ & $106(73,6)$ & $38(26,4)$ & $302(91,5)$ & $28(8,5)$ \\
\hline$p^{a}$ & \multicolumn{4}{|c|}{$\leq 0,001^{*}$} & & \\
\hline \multicolumn{7}{|l|}{ MM } \\
\hline normal & $272(66,7)$ & $44(66,7)$ & $72(67,9)$ & $24(63,2)$ & $200(66,2)$ & $20(71,4)$ \\
\hline baixa & $136(33,3)$ & $22(33,3)$ & $34(32,1)$ & $14(36,8)$ & $102(33,8)$ & $08(28,6)$ \\
\hline$p^{b}$ & \multicolumn{2}{|c|}{1,000} & \multicolumn{2}{|c|}{0,593} & \multicolumn{2}{|l|}{0,576} \\
\hline \multicolumn{7}{|l|}{ Força } \\
\hline normal & $283(69,4)$ & $33(50,0)$ & $73(68,9)$ & $23(60,5)$ & $210(69,5)$ & $10(35,7)$ \\
\hline baixa & $125(30,6)$ & $33(50,0)$ & $33(31,1)$ & $15(39,5)$ & $92(30,5)$ & $18(64,3)$ \\
\hline$p^{b}$ & & & & & $\leq 0,001^{*}$ & \\
\hline Atividade Física $r$ & er e Locomoc & & & & & \\
\hline Total & 336 & $0,9)$ & & $8,8)$ & & $93(28,2)$ \\
\hline$p^{a}$ & & & & & & \\
\hline MM & & & & & & \\
\hline normal & 222 & $6,1)$ & $69(69,7)$ & $27(60,0)$ & $153(64,6)$ & $67(72,0)$ \\
\hline baixa & 114 & $3,9)$ & $30(30,3)$ & $18(40,0)$ & $84(35,4)$ & $26(38,0)$ \\
\hline$p^{b}$ & & & & & & \\
\hline Força & & & & & & \\
\hline normal & $228(67,8)$ & $88(63,8)$ & $71(71,7)$ & $25(55,6)$ & $157(66,2)$ & $63(67,7)$ \\
\hline baixa & $108(32,2)$ & $50(36,2)$ & $28(28,3)$ & $20(44,4)$ & $80(33,8)$ & $30(32,3)$ \\
\hline$p^{b}$ & & & & & & \\
\hline
\end{tabular}

Notas: MM=massa muscular; a=análise do qui-quadrado (e nível de significância) entre nível de atividade física e sexo; b=análise do qui-quadrado (e nível de significância) entre nível de atividade física e categoria de massa e força muscular. ${ }^{*} p<0,05$

$\mathrm{Na}$ análise de regressão logística, verificou-se que indivíduos com baixo nível de atividade física nos domínios ocupacional e, exercício físico no lazer e AFH têm apro- 
ximadamente de duas a três vezes mais chances de apresentarem baixa FM $(\mathrm{p}<0,05)$ quando comparados aos seus pares mais ativos, respectivamente. $\mathrm{Na}$ análise múltipla ajustada por grupo etário e sexo tal associação permanece para o domínio exercício físico no lazer e AFH, aproximadamente 2 vezes mais chances (Tabela 4).

TABELA 3 - Frequência dos participantes segundo nível de atividade física habitual, condição de massa e força muscular para amostra geral e segundo o sexo.

\begin{tabular}{|c|c|c|c|c|c|c|}
\hline \multicolumn{7}{|c|}{ Atividade Física Habitual } \\
\hline & \multicolumn{2}{|c|}{ Amostra geral } & \multicolumn{2}{|c|}{ Homens } & \multicolumn{2}{|c|}{ Mulheres } \\
\hline & Maior & Menor & Maior & Menor & Maior & Menor \\
\hline Categorias & n (\%) & n (\%) & n (\%) & n (\%) & n (\%) & $n(\%)$ \\
\hline Total & $361(76,2)$ & $113(23,8)$ & $108(75,0)$ & $36(25,0)$ & $253(76,7)$ & $77(23,3)$ \\
\hline $\mathrm{p}^{\mathrm{a}}$ & & & \multicolumn{4}{|c|}{0,695} \\
\hline \multicolumn{7}{|l|}{ MM } \\
\hline normal & $268(74,2)$ & $88(77,9)$ & $81(75,0)$ & $27(75,0)$ & $187(73,9)$ & $61(79,2)$ \\
\hline baixa & $93(25,8)$ & $25(22,1)$ & $27(25,0)$ & $9(25,0)$ & $66(26,1)$ & $16(20,8)$ \\
\hline$p^{b}$ & \multicolumn{2}{|c|}{0,435} & \multicolumn{2}{|c|}{1,000} & \multicolumn{2}{|c|}{0,345} \\
\hline \multicolumn{7}{|l|}{ Força } \\
\hline normal & $282(78,1)$ & $75(66,4)$ & $86(79,6)$ & $22(61,1)$ & $196(77,5)$ & $53(68,8)$ \\
\hline baixa & $79(21,9)$ & $38(33,6)$ & $22(20,4)$ & $14(38,9)$ & $57(22,5)$ & $24(31,2$ \\
\hline$p^{b}$ & \multicolumn{2}{|c|}{$0,012^{*}$} & \multicolumn{2}{|c|}{$0,026^{*}$} & \multicolumn{2}{|c|}{0,123} \\
\hline
\end{tabular}

Notas: $M M=$ =massa muscular; a=análise do qui-quadrado (e nível de significância) entre nível de atividade física e sexo; b=análise do qui-quadrado (e nível de significância) entre nível de atividade física e categoria de massa e força muscular. ${ }^{*} p<0,05$.

Quando analisado segundo sexo, homens com baixo nível de atividade física no domínio ocupacional e AFH, e mulheres com baixo nível de atividade física no domínio exercício físico no lazer apresentaram maior risco para baixa FM, quando comparados aos seus pares mais ativos $(\mathrm{p}<0,05)$, sendo esta associação válida para o modelo de regressão univariado ou ajustado por grupo etário (Tabela 4).

TABELA 4 - Modelos de regressão logística univariado e múltiplo da associação entre nível de atividade física habitual e força muscular dos participantes.

\begin{tabular}{lcccc}
\hline \multirow{2}{*}{ Baixo Nível AF } & \multicolumn{2}{c}{ Não ajustado } & \multicolumn{2}{c}{ Ajustado* $^{*}$} \\
\cline { 2 - 5 } & OR & IC 95\% & OR & IC 95\% \\
\hline Ocupacional & 1,65 & $1,10-2,49^{*}$ & 1,51 & $0,99-2,31$ \\
\hline Homens & 2,47 & $1,18-5,16^{*}$ & 2,20 & $1,02-4,74^{*}$ \\
\hline Mulheres & 1,38 & $0,84-2,27$ & 1,29 & $0,78-2,14$ \\
\hline Lazer & 2,26 & $1,34-3,83^{*}$ & 2,38 & $1,36-4,17^{*}$ \\
\hline Homens & 1,44 & $0,67-3,11$ & 1,53 & $0,68-3,44$ \\
\hline Mulheres & 4,11 & $1,83-9,24^{*}$ & 4,02 & $1,77-9,14^{*}$ \\
\hline Lazer e locomoção & 1,20 & $0,79-1,82$ & 1,19 & $0,78-1,82$ \\
\hline Homens & 2,03 & $0,97-4,22$ & 1,92 & $0,89-4,10$ \\
\hline Mulheres & 0,93 & $0,56-1,57$ & 0,95 & $0,56-1,59$ \\
\hline Habitual & 1,89 & $1,26-2,84^{*}$ & 1,72 & $1,13-2,61^{*}$ \\
\hline Homens & 2,76 & $1,33-5,73^{*}$ & 2,40 & $1,12-5,17^{*}$ \\
\hline Mulheres & 1,60 & $0,98-2,61$ & 1,49 & $0,90-2,46$ \\
\hline
\end{tabular}

Notas: * Regressão logística ajustada por sexo e grupo etário, com a amostra total, e por grupo etário, com a amostra separada por sexo; OR=odds ratio; IC 95\%=intervalo de confiança. 
$\mathrm{Na}$ análise de associação entre o nível de atividade física e FM, foi verificado que a variável de ajuste idade apresentou significância estatística nos modelos de regressão logística múltiplos, com maior chance para indivíduos com 70 anos ou mais.

\section{Discussão}

Os principais achados deste estudo foram que após avaliar a prática de atividade física em diferentes domínios (ocupacional, exercício físico no lazer e locomoção) e AFH foi observado que idosos insuficientemente ativos tendem a ter mais chances de apresentarem baixa FM.

Maiores valores de FM e MM foram identificados nesse estudo para pessoas do sexo masculino, em relação ao sexo feminino $(\mathrm{p}<0,05)$. Estudos prévios também observaram diferenças significativas na FM entre os sexos ${ }^{4,5}$. Em relação à $\mathrm{MM}$, os valores medianos apresentados no presente estudo (IMM $=10,5 \mathrm{~kg} / \mathrm{m}^{2}$ para homens e $8,5 \mathrm{~kg} / \mathrm{m}^{2}$ para mulheres) estão em conformidade com outro estudo realizado com amostra representativa na maior cidade do Brasil ${ }^{3}$.

De fato, homens têm maior quantidade de MM e maior FM, em relação às mulheres, principalmente nos grupos etários adultos jovens e idosos, o que pode ser um dos fatores que auxiliaram explicar as maiores taxas de sarcopenia nas mulheres $^{2}$. Contudo o declínio da MM pode ser mais acentuado para os homens ${ }^{2,3,16}$, e este declínio pode ser causa de maior incapacidade funcional. Ao longo do envelhecimento, idosos do sexo masculino têm maior redução da MM e da FM em função das alterações hormonais (declínio do hormônio do crescimento, do fator de crescimento relacionado à insulina, e da testosterona). Entretanto mesmo tendo os homens maior redução da MM e FM mediante as possíveis causas supracitadas, as mulheres idosas, podem apresentar maior prevalência de limitações funcionais ${ }^{17}$.

Quando analisado os escores de AFH e os seus diferentes domínios, não foi verificado diferenças entre os sexos $(p>0,05)$ para os domínios da atividade física no exercício no lazer e na AFH. Todavia, homens apresentaram maiores escores para o domínio atividade física no lazer e locomoção, enquanto as mulheres apresentaram maiores valores para o domínio ocupacional $(\mathrm{p}<0,05)$. Nossos achados corroboram aos encontrados previamente ${ }^{10,18}$, uma vez que por fatores de segurança e culturais os homens acabam sendo mais ativos nas atividades de locomoção e as mulheres ainda são as principais responsáveis pelas tarefas domésticas.

Quando observada a proporção de indivíduos que apresentam baixo nível de atividade física, não foi verificada diferenças entre os sexos $(p>0,05)$ para os domínios da atividade física ocupacional, atividade física no lazer e locomoção (Tabela 2) e AFH (Tabela 3). Em contrapartida, no domínio exercício físico no lazer, o sexo masculino apresentou maior percentual de indivíduos com baixo nível de atividade física. Diferentemente, do resultado apresentado por Codogno et al. ${ }^{19}$, o qual aponta maior proporção da prática de atividade física nesse domínio para homens em pessoas atendidas pelo sistema de saúde pública. Tal fato pode ter ocorrido devido adoção do ponto de corte do presente estudo (< tercil 1), que foi utilizado com o intuito de selecionar as pessoas que apresentavam nível de atividade física extremamente baixo.

Nesse estudo apenas a baixa FM foi associada com o baixo nível de AFH, em especial, para o sexo masculino. Em relação aos diferentes domínios da pratica de atividade física foi verificado que o baixo nível de atividade física associou-se a baixa FM no domínio ocupacional para amostra total (contemplando ambos os sexos na mesma amostra) e para o sexo masculino. $O$ estudo de seguimento (22 anos) de Sternang et al. ${ }^{20}$ com amostra de indivíduos com idade superior a 50 
anos revelou que menor declínio na FM em idade mais avançada está associado a menor execução de atividade laboral extenuante na vida adulta jovem. De fato, a execução prolongada e repetitiva de atividades com essas características no trabalho podem causar prejuízos funcionais. Contudo, em nosso estudo as atividades desenvolvidas pelos idosos no trabalho apresentam intensidade leve (70\%). Assim, nesse domínio o baixo nível de atividade física associou-se a baixa FM.

A associação entre baixa FM e baixo nível de atividade física foi observada no domínio exercício físico no lazer tanto para amostra total quanto para o sexo feminino. Tal evidência pode ter sido observada devido ao fato das atividades contidas nesta sessão estarem relacionadas às atividades esportivas e treinamento resistido em academias, que consistem em atividades comprovadamente mais eficazes para a manutenção e aumento da $\mathrm{FM}^{21,22}$. Mesmo sendo observado em alguns estudos que os homens são mais adeptos a essas atividades ${ }^{9,10}$, a diferença entre sexos, verificada no presente estudo, ocorreu para o sexo feminino, em parte em função de no envelhecimento a adesão dessas atividades pelos homens diminuir ${ }^{10,23}$.

Evidências confirmam que a prática de atividade física está associada ao aumento e manutenção da $\mathrm{MM}^{24-26}$. Entretanto, não foi verificada associação entre baixa MM e baixo nível de atividade física habitual total e em seus diferentes domínios em nosso estudo. Silva et al..$^{27}$ aplicaram treinamento com pesos em mulheres com idade igual ou superior a 50 anos por 12 semanas e após esse período não verificaram mudança na MM, somente o aumento da FM em todos os grupos musculares investigados. Dessa forma, infere-se que outros fatores comportamentais como, a ingestão adequada de nutrientes, especialmente, proteínas, adicionado a pratica de atividade física com maior intensidade podem estar mais relacionados à manutenção e ao aumento da $\mathrm{MM}$ em indivíduos mais velhos ${ }^{28}$.

A análise de regressão logística reforçou a associação entre AFH e FM. Neste sentido, pode-se concluir que baixa prática de AFH aumentam as chances de menor FM, sobretudo para os homens. A partir dos resultados expostos, é possível destacar a importância da FM para o envelhecimento, a considerar pela redução média ao longo dos grupos etários, apresentadas por diferentes estudos ${ }^{4,5}$, pelas maiores diferenças nestas reduções, comparadas à $\mathrm{MM}$, e pelas diferenças entre os sexos, que pode ser determinante na funcionalidade do idoso.

Além disso, reduções na MM promove decréscimo na FM, e em consequência, outras perdas qualitativas metabólicas e fisiológicas, como a taxa metabólica basal, o consumo máximo e oxigênio e o gasto energético total. Esses fatores associados à possibilidade de um quadro de morbidade, desnutrição e em função do próprio envelhecimento, potencializa não apenas a redução da MM, mas também a $\mathrm{FM}^{29}$. Este desfecho pode contribuir para maior incapacidade funcional, inatividade física, sedentarismo (comportamentos com gasto energético inferior a 1,5METs), dependência, baixa qualidade de vida e maior risco para morte.

Como limitações o presente estudo apresenta a sua característica transversal, o que não permite o estabelecimento de relação de causa e efeito, ou seja, menores valores de AFH proporcionam menores valores de FM, bem como, se em uma análise longitudinal, a redução da MM poderia apresentar maior relação com a redução dos escores de atividade física. O método duplamente indireto de avaliação da MM também foi um fator limitante do presente estudo. Como aspectos positivos destaca-se o ineditismo de se verificar a relação entre os diferentes domínios de atividade física na FM e MM de usuários do sistema de saúde brasileiro. Ressalta-se também que o questionário utilizado para a avaliação no presente estudo além de ser validado para a população brasileira ${ }^{15}$, também foi testado contra métodos padrão ouro, 
como a água duplamente marcada, para avaliação da prática de atividade física ${ }^{30}$.

A partir dos achados desse estudo, apresenta-se como aplicações práticas, a importância do incentivo da prática de atividade física e serviços de manutenção da saúde por profissionais nas UBS, almejando-se a redução de internações futuras nessa população oriundas por decréscimo da FM e MM.

Em conclusão, homens e mulheres usuários dos serviços de saúde pública apresentaram níveis semelhantes de atividade física habitual, entretanto, apenas a FM apresentou-se associada ao nível de atividade física, com os homens com baixo nível de atividade física habitual apresentando maior risco para baixa FM, em comparação àqueles aos mais ativos.

\section{Contribuição dos autores}

Santos VR auxiliou na coleta de dados, análise e interpretação dos dados, redação do manuscrito, e revisão crítica. Macedo DO e Nascimento CB auxiliaram na coleta de dados e redação do manuscrito. Christofaro DGD redação do manuscrito. Gobbo LA aquisição dos sujeitos, coleta de dados, elaboração do manuscrito, análise e interpretação dos dados, redação e revisão crítica do manuscrito.

\section{Agradecimentos}

Ao Grupo de Estudo em Saúde, Atividade Física e Economia (GESAFE - FCT/UNESP).

\section{Referências}

1. World Health Organization (WHO). Active ageing. A policy framework; 2002. [citado 2014 jan 14]. Disponível em: http://whqlibdoc.who.int/hq/2002/WHO_NMH_NPH_02.8.pdf.

2. Janssen I, Heymsfield SB, Wang ZM, Ross RR. Skeletal muscle mass and distribution in 468 men and women aged 18-88 yr. J Appl Physiol. 2000; 89(1): 81-8.

3. Gobbo LA, Dourado DAQS, Almeida MF, Duarte YAO, Lebrão ML, Marucci MF. Skeletalmuscle mass of São Paulo city elderly - SABE Survey: Health, Well-being and Aging. Rev. Bras. Cineantropom. Desempenho Hum. 2012; 14(1): 1-10.

4. Schlussel MM, Anjos LA, Vasconcellos MTL, Kac G. Reference values of handgrip dynamometry of healthy adults: A population-based study. Clin Nutr. 2008; 27(4): 601-7.

5. Perna FM, Coa K, Troiano RP, Lawman HG, Wang CY, Li Y, et al. Muscular Grip Strength Estimates of the U.S. Population from the National Health and Nutrition Examination Survey 2011-2012. J Strength Cond Res. 2016; 30(3):867-74.

6. Mazzeo RS, Cavanagh P, Evans WJ, Fiatarone MA, Hagberg J, McAuley E, et al. Exercício e atividade física para pessoas idosas: Colégio Americano de Medicina Esportiva, posicionamento oficial. Rev Bras Ativ Fis Saude. 1998; 3(1): 48-78.

7. Janssen I, Heymsfield SB, Ross R. Low relative skeletal muscle mass (sarcopenia) in older persons is associated with functional impairment and physical disability. J Am Geriatr Soc. $2002 ;$ 50(5): 889-96.

8. Matsudo SM, Matsudo VKR, Barros Neto TL. Atividade física e envelhecimento: aspectos epidemiológicos. Rev Bras Med Esporte. 2001; 7(1): 2-13.

9. Florindo AA, Hallal PC, Moura EC, Malta DC. Practice of physical activities and associated factors in adults, Brazil, 2006. Rev. Saúde Pública. 2009; 43 (Suppl 2): 1-8.

10. Mourao ARC, Novais FV, Andreoni S, Ramos LR. Atividade física de idosos relacionada ao transporte e lazer, Maceió, Brasil. Rev. Saúde Pública. 2013; 47(6): 1112-22.

11. Instituto Brasileiro de Geografia e Estatística (IBGE). Pesquisa Nacional de Saúde 2013: Percepção do estado de saúde, estilos de vida e doenças crônicas, Brasil, grandes regiões e unidades da federação; 2014. [citado 2016 jun 27]. Disponível em: http://www.ibge.gov. br/home/estatistica/populacao/pns/2013/default.shtm.

12. Lee RC, Wang Z, Heo M, Ross R, Janssen I, Heymsfield SB. Total-body skeletal muscle mass: development and cross-validation of anthropometric prediction models. Am J Clin Nutr. 2000; 72(3): 796-803.

13. Lohman TG, Roche AF, Martorell R. Anthropometric standardization reference manual: 
Stature, recumbent length, and weight. Champaign, Illinois: Human Kinetics Books, 1988.

14. Baecke JA, Burema J, Frijters JE. A short questionnaire for the measurement of habitual physical activity in epidemiological studies. Am J Clin Nutr. 1982; 36(5): 936-42.

15. Florindo AA, Latorre MRDO, Jaime OC, Tanaka T, Zerbini CAF. Methodology to evaluation the habitual physical activity in men aged 50 years or more. Rev. Saúde Pública. 2004; 38(2): 307-14.

16. Lauretani F, Russo CR, Bandinelli S, Bartali B, Cavazzini C, Di Iorio A, et al. Age associated changes in skeletal muscles and their effect on mobility: an operational diagnosis of sarcopenia. J Appl Physiol. 2003; 95(5): 1851-60.

17. Roubenoff R, Hughes VA. Sarcopenia: current concepts. J Gerontol A Biol Sci Med Sci. 2000; 55(12): M716-24.

18. Codogno JS, Turi BC, Fernandes RA, Monteiro HL. Comparação de gastos com serviços de atenção básica à saúde de homens e mulheres em Bauru, São Paulo, 2010. Epidemiol. Serv. Saúde. 2015; 24(1): 115-22.

19. Codogno JS, Turi BC, Fernandes RA, Monteiro HL. Nível de atividade física no lazer em usuários do sistema único de saúde. Rev Bras Ativ Fis Saude. 2012; 17(6): 543-51.

20. Sternang O, Reynolds CA, Finkel D, Ernsth-Bravell M, Pedersen NL, Dahl Aslan AK. Factors associated with grip strength decline in older adults. Age Ageing. 2015; 44(2): 269-74.

21. Melov S, Tarnopolsky MA, Beckman K, Felkey K, Hubbard A. Resistance exercise reverses aging in human skeletal muscle. PLoS One. 2007; 2(5): e465.

22. Geirsdottir OG, Arnarson A, Ramel A, Briem K, Jonsson PV, Thorsdottir I. Muscular strength and physical function in elderly adults 6-18 months after a 12-week resistance exercise program. Scand J Public Health. 2015; 43(1): 76-82.

23. Zanchetta LM, Barros MBA, César CLG, Carandina L, Goldbaum M, Alves MCGP. Inatividade física e fatores associados em adultos, São Paulo, Brasil. Rev. Bras. Epidemiol. 2010; 13(3): 387-99.

24. Park H, Park S, Shephard RJ, Aoyagi Y. Yearlong physical activity and sarcopenia in older adults: the Nakanojo Study. Eur J Appl Physiol. 2010; 109(5): 953-61.

25. Shephard RJ, Park H, Park S, Aoyagi Y. Objectively measured physical activity and progressive loss of lean tissue in older Japanese adults: longitudinal data from the Nakanojo study. J Am Geriatr Soc. 2013; 61(11): 1887-93.

26. Bann D, Kuh D, Wills AK, Adams J, Brage S, Cooper R. Physical activity across adulthood in relation to fat and lean body mass in early old age: findings from the medical research council national survey of health and development, 1946-2010. Am J Epidemiol. 2014; 179(10): 1197-207.

27. Silva CM, Gurjão ALD, Ferreira L, Gobbi LTB, Gobbi S. Efeito do treinamento com pesos, prescritos por zona de repetições máximas, na força muscular e composição corporal em idosas. Rev. Bras. Cineantropom. Desempenho Hum. 2006; 8(4): 39-45.

28. Strandberg E, Edholm P, Ponsot E, Wåhlin-Larsson B, Hellmén E, Nilsson A, et al. Influence of combined resistance training and healthy diet on muscle mass in healthy elderly women: a randomized controlled trial. J Appl Physiol (1985). 2015; 119(8): 918-25.

29. Fried LP, Tangen CM, Walston J, Newman AB, Hirsch C, Gottdieneer J, et al. Frailty in older adults: evidence for a phenotype. J Gerontol Med Sci. 2001; 56(3): M146-56.

30. Philippaerts RM, Westerterp KR, Lefevre J. Doubly labelled water validation of three physical activity questionnaires. Int J Sports Med 1999; 20: 284-89.

ENDEREÇO PARA

CORRESPONDÊNCIA

LUÍS ALBERTO GOBBO

luisgobbo@fct.unesp.br
Rua Roberto Simonsen, 305, Centro

Educacional, CEP 19060-080,

Presidente Prudente, SP,

Telefone + 551832295720

$\mathrm{fax}+551832295710$
RECEBIDO

REVISADO

APROVADO
22/12/2015

$05 / 07 / 2016$

$16 / 07 / 2016$ 